\title{
一类集值映射的传递性、 混合性与混沌*
}

\author{
廖公夫 $^{* *}$ \\ (吉林大学数学学院, 长春 130012) \\ 张玉成 \\ (中国科学技术大学数学系, 合肥 230026)
}

王立冬

(大连民族学院应用数理系, 大连 116600)

\begin{abstract}
摘要 考虑连续映射 $f: X \rightarrow X$ 以及 $f$ 诱导的 $\mathcal{K}(X)$ 到自身的连续映射 $\bar{f}$, 其 中 $X$ 为度量空间, $\mathcal{K}(X)$ 为 $X$ 的所有非空紧子集赋予 Hausdorff 度量所得空间. 针对 Román-Flores 提出的 $f$ 混沌是否蕴涵 $\bar{f}$ 混沌以及 Fedeli 提出的什么时候 $f$ 混沌蕴涵 $\bar{f}$ 混沌的问题, 探讨 $f$ 与 $\bar{f}$ 的传递、弱混合和混合等相关动力性质之 间的关系，并应用所得结果对 Román-Flores 的问题和 Fedeli 的问题给出了满意的 回答.
\end{abstract}

\section{关键词 集值映射 传递性 弱混合性 混合性 混沌}

本文总假设 $f$ 为度量空间 $(X, d)$ 到自身的连续映射, $\bar{f}$ 为 $f$ 诱导的集值映 射, 亦即 $f$ 到 $\mathcal{K}(X)$ 的自然扩张, 其中 $\mathcal{K}(X)$ 为 $X$ 的所有非空紧子集赋予由 $d$ 诱导的 Hausdorff 度量所得空间. 众所周知, 弄清 $X$ 中的点如何运动是研究系统 $(X, f)$ 的主要任务. 然而, 在生命起源、人口统计学、数值模拟、吸引子等许多 领域或问题中, 仅知道 $X$ 中的点如何运动还很不够 ${ }^{[1,2]}$, 人们还需要知道 $X$ 中的 子集怎样运动, 因此必须研究与 $(X, f)$ 相关的集值动力系统 $(\mathcal{K}(X), \bar{f})$.

本文的目的是研究 $f$ 与 $\bar{f}$ 的传递性、混合性以及混沌性之间的关系. 一个 映射是混沌的意指它具有 Devaney 定义中的如下 3 条性质: (1) 传递性，(2) 周 期稠密性，(3) (对初值的) 敏感性 ${ }^{[3]}$.

* 国家自然科学基金 (批准号: 19971035) 和吉林大学创新基金资助项目

** E-mail: liaogf@email.jlu.edu.cn 
由于当系统含无限多点时, Devaney 定义中的性质 (1) 和 (2) 蕴涵 (3) (参见文 献 [4 6]), 因此为确定一个映射是否混沌, 只需考察它是否具有传递性和周期稠 密性就够了. 读者在文献 [7 9] 中会发现传递性和周期稠密性不仅蕴涵敏感性, 而且还蕴涵着更严厉甚至是出人意料的混沌性态.

Román-Flores $^{[10]}$ 提出如下问题:

(Q1) $f$ 混沌是否蕴涵 $\bar{f}$ 混沌?

为解决此问题他以圆周的无理旋转为例说明 $f$ 传递未必蕴涵 $\bar{f}$ 传递 ${ }^{[10]}$. 由 于圆周的无理旋转并非混沌 (无周期点), 易见问题 (Q1) 没有解决. 此后, Fedeli ${ }^{[11]}$ 提出

(Q2) 什么时候 $f$ 混沌蕴涵 $\bar{f}$ 混沌?

Fedeli 本人的回答是: $f$ 周期稠密且 $\bar{f}$ 传递便可知 $\bar{f}$ 混沌 ${ }^{[11]}$. 这个回答显然 也是不能令人满意的, 因为仅从 $f$ 混沌本身还无法断定 $\bar{f}$ 是否传递.

本文的工作正是为解决问题 $(\mathrm{Q} 1)$ 和 (Q2) 而展开的.

\section{1 预备}

称 $f$ 为 (拓扑) 传递的, 如果对 $X$ 中任意非空开集 $U$ 和 $V$, 存在正整数 $n$, 使得 $f^{n}(U) \cap V \neq \emptyset$; 称 $f$ 为 (拓扑) 弱混合的, 如果两个 $f$ 的 Descartes 积 $f \times f$ 是传递 的, 亦即对 $X$ 中任意非空开集 $U_{1}, U_{2}, V_{1}$ 和 $V_{2}$, 存在正整数 $n$, 使得 $f^{n}\left(U_{i}\right) \cap V_{i} \neq$ $\emptyset, i=1,2 . m$ 个 $f$ 的 Descartes 积简单地记作 $f_{m}$. 称 $f$ 为 (拓扑) 混合的, 如果对 $X$ 中任意非空开集 $U$ 和 $V$, 存在正整数 $N$, 使得 $f^{n}(U) \cap V \neq \emptyset$ 对所有的 $n \geqslant N$ 都成立.

显然，混合的映射是弱混合的，弱混合的映射是传递的.

如前所述, $\mathcal{K}(X)$ 表示由 $X$ 中所有非空紧子集构成的集族. 令 $H$ 表示 $\mathcal{K}(X)$ 上的 Hausdorff 度量, 它定义为: 对任意 $A, B \in \mathcal{K}(X)$,

$$
H(A, B)=\max \{\operatorname{dist}(A, B), \operatorname{dist}(B, A)\},
$$

其中 $\operatorname{dist}(A, B)=\sup _{a \in A} \inf _{b \in B} d(a, b)$.

值得注意的是 $\mathcal{K}(X)$ 上由 Hausdorff 度量诱导的拓扑就是 $\mathcal{K}(X)$ 上的 Vietoris 拓扑，而 Vietoris 拓扑的基中的每个成员形如

$$
\mathcal{B}\left(U_{1}, \cdots, U_{n}\right)=\left\{F \in \mathcal{K}(X) \mid F \subset \cup_{i=1}^{n} U_{i}, F \cap U_{i} \neq \emptyset, i=1, \cdots, n\right\},
$$

其中 $U_{1}, \cdots, U_{n}$ 为 $X$ 的非空开集 (参见文献 [12] p. 8, 不过请注意那里误把基说成 了子基).

$f$ 到 $\mathcal{K}(X)$ 的扩张 $\bar{f}$ 定义为: 对任意 $A \in \mathcal{K}(X), \bar{f}(A)=\{f(a) \mid a \in A\}$. 不难 证明 $f$ 连续蕴涵 $\bar{f}$ 连续. 我们用 $B(A, \varepsilon)$ 表示集合 $\{F \in \mathcal{K}(X) \mid H(A, F)<\varepsilon\}$.

下述引理 1.1 和 1.2 是明显的:

引理 $1.1 f$ 传递当且仅当对 $X$ 中任意非空开集 $U$ 和 $V$, 存在正整数 $n$, 使 得 $f^{-n}(U) \cap V \neq \emptyset$.

引理 $1.2 f$ 混合当且仅当对任意 $m>0, f_{m}$ 混合. 


\section{引理 1.3 下述论断等价:}

(1) $f$ 弱混合,

(2) 对任意 $m \geqslant 2, f_{m}$ 传递,

(3) 对 $X$ 中任意非空开集 $U$ 和 $V$, 存在 $n>0$, 使得

$$
f^{n}(U) \cap V \neq \emptyset, \quad f^{n}(V) \cap V \neq \emptyset .
$$

证 (1) $\Rightarrow(2)$. 设 $f$ 弱混合. 根据定义, $f_{2}$ 传递. 假设对某个 $k \geqslant 2$, 已经知 道 $f_{k}$ 传递. 设 $U_{1}, U_{2}, \cdots, U_{k}, U_{k+1}, V_{1}, V_{2}, \cdots, V_{k}, V_{k+1}$ 是 $X$ 中任意 $2(k+1)$ 个 非空开集. 因为 $f_{2}$ 传递, 根据引理 1.1 , 知存在 $l>0$, 使得

$$
U=U_{k} \cap f^{-l}\left(U_{k+1}\right), \quad V=V_{k} \cap f^{-l}\left(V_{k+1}\right)
$$

都是非空开集. 因为 $f_{k}$ 传递, 故存在 $n>0$, 使得

$$
f^{n}\left(U_{i}\right) \cap V_{i} \neq \emptyset, \quad f^{n}(U) \cap V \neq \emptyset, \quad i=1,2, \cdots, k-1 .
$$

注意到

$$
f^{n}\left(U_{k}\right) \cap V_{k} \supset f^{n}(U) \cap V \neq \emptyset
$$

以及

$$
f^{n}\left(U_{k+1}\right) \cap V_{k+1} \supset f^{n}\left(f^{l}(U)\right) \cap f^{l}(V) \supset f^{l}\left(f^{n}(U) \cap V\right) \neq \emptyset,
$$

得知 $f_{k+1}$ 传递. 据归纳法, 知结论成立.

(2) $\Rightarrow$ (3). 显然.

(3) $\Rightarrow(1)$. 设 $U_{1}, U_{2}, V_{1}, V_{2}$ 是 $X$ 的非空开集. 首先由于易见 $f$ 传递, 故据引 理 1.1 , 知存在 $n_{1}>0$, 使得

$$
A=V_{1} \cap f^{-n_{1}}\left(V_{2}\right) \neq \emptyset,
$$

又存在 $n_{2}>0$, 使得

$$
B=f^{-n_{2}}(A) \cap f^{-n_{1}}\left(U_{2}\right) \neq \emptyset .
$$

其次对非空开集 $B$ 和 $U_{1}$, 存在 $n_{3}>0$, 使得

$$
f^{n_{3}}(B) \cap B \neq \emptyset, \quad f^{n_{3}}\left(U_{1}\right) \cap B \neq \emptyset .
$$

最后令 $n=n_{2}+n_{3}$, 有

$$
\begin{aligned}
& f^{n}\left(U_{1}\right) \cap V_{1}=f^{n_{2}+n_{3}}\left(U_{1}\right) \cap V_{1} \\
\supset & f^{n_{2}+n_{3}}\left(U_{1}\right) \cap f^{n_{2}} f^{-n_{2}}(A) \supset f^{n_{2}}\left(f^{n_{3}}\left(U_{1}\right) \cap f^{-n_{2}}(A)\right) \\
\supset & f^{n_{2}}\left(f^{n_{3}}\left(U_{1}\right) \cap B\right) \neq \emptyset .
\end{aligned}
$$

由于

$$
\begin{aligned}
& f^{-n_{1}} f^{-n_{2}}\left(f^{n_{2}+n_{3}}\left(U_{2}\right) \cap V_{2}\right) \supset f^{-n_{1}} f^{n_{3}}\left(U_{2}\right) \cap f^{-\left(n_{1}+n_{2}\right)}\left(V_{2}\right) \\
\supset & f^{n_{3}} f^{-n_{1}}\left(U_{2}\right) \cap f^{-\left(n_{1}+n_{2}\right)}\left(V_{2}\right) \supset f^{n_{3}}(B) \cap B \neq \emptyset,
\end{aligned}
$$

还有 $f^{n}\left(U_{2}\right) \cap V_{2} \neq \emptyset$, 因此 $f$ 弱混合. 


\section{2 传递性、混合性与 Fedeli 问题的回答}

定理 2.1 下述条件等价:

(i) $f$ 弱混合,

(ii) $\bar{f}$ 弱混合,

(iii) $\bar{f}$ 传递.

证 (i) $\Rightarrow$ (ii). 设 $f$ 弱混合, $\mathcal{U}, \mathcal{V}$ 为 $\mathcal{K}(X)$ 的非空开集，则存在 $X$ 的非 空开集 $U_{1}, U_{2}, \cdots, U_{s}$ 以及 $V_{1}, V_{2}, \cdots, V_{t}$, 使得 $\mathcal{B}_{1}=\mathcal{B}\left(U_{1}, \cdots, U_{s}\right) \subset \mathcal{U}, \mathcal{B}_{2}=$ $\mathcal{B}\left(V_{1}, \cdots, V_{t}\right) \subset \mathcal{V}$. 令 $m=\max \{s, t\}$, 再令

$$
\begin{gathered}
\bar{U}_{i}= \begin{cases}U_{i}, & 1 \leqslant i \leqslant s, \\
U_{s}, & s \leqslant i \leqslant m, \\
V_{i}, & m+1 \leqslant i \leqslant m+t, \\
V_{t}, & m+t \leqslant i \leqslant 2 m,\end{cases} \\
\bar{V}_{i}= \begin{cases}V_{i}, & 1 \leqslant i \leqslant t \text { 或 } m+1 \leqslant i \leqslant m+t, \\
V_{t}, & t \leqslant i \leqslant m \text { 或 } m+t \leqslant i \leqslant 2 m,\end{cases}
\end{gathered}
$$

易见, $\bar{U}_{i}$ 和 $\bar{V}_{i}$ 都是 $X$ 的非空开集. 因为 $f$ 弱混合，据引理 1.3, $f_{2 m}$ 传递. 因此 存在 $n>0$, 使得当 $1 \leqslant i \leqslant 2 m$ 时, $f^{n}\left(\bar{U}_{i}\right) \cap \bar{V}_{i} \neq \emptyset$. 于是对每个 $i=1,2, \cdots, 2 m$, 可取 $x_{i} \in \bar{U}_{i}, y_{i} \in \bar{V}_{i}$, 使得 $f^{n}\left(x_{i}\right)=y_{i}$. 置

$$
\begin{array}{cl}
E_{1}=\left\{x_{1}, x_{2}, \cdots, x_{m}\right\}, & F_{1}=\left\{y_{1}, y_{2}, \cdots, y_{m}\right\}, \\
E_{2}=\left\{x_{m+1}, x_{m+2}, \cdots, x_{2 m}\right\}, \quad F_{2}=\left\{y_{m+1}, y_{m+2}, \cdots, y_{2 m}\right\},
\end{array}
$$

我们有 $\bar{f}^{n}\left(E_{i}\right)=F_{i}, i=1,2$. 由于 $E_{1} \in \mathcal{B}_{1}$, 而 $E_{2}, F_{1}, F_{2} \in \mathcal{B}_{2}$, 因此有

$$
\bar{f}^{n}(\mathcal{U}) \cap \mathcal{V} \supset \bar{f}^{n}\left(\mathcal{B}_{1}\right) \cap \mathcal{B}_{2} \neq \emptyset, \quad \bar{f}^{n}(\mathcal{V}) \cap \mathcal{V} \supset \bar{f}^{n}\left(\mathcal{B}_{2}\right) \cap \mathcal{B}_{2} \neq \emptyset .
$$

据引理 $1.3, \bar{f}$ 为弱混合.

(ii) $\Rightarrow$ (iii). 显然.

(iii) $\Rightarrow$ (i). 设 $\bar{f}$ 传递, $U$ 和 $V$ 为 $X$ 的非空开集. 令 $\mathcal{U}=\mathcal{B}(U, V), \mathcal{V}=\mathcal{B}(V)$, 则 $\mathcal{U}$ 和 $\mathcal{V}$ 为 $\mathcal{K}(X)$ 的非空开集. 于是存在 $n>0$, 使得 $\bar{f}^{n}(\mathcal{U}) \cap \mathcal{V} \neq \emptyset$. 亦即 存在 $K \in \mathcal{U}, F \in \mathcal{V}$, 使得 $\bar{f}^{n}(K)=F$. 任取 $x \in K \cap U, y \in K \cap V$, 我们有 $f^{n}(x) \in V, f^{n}(y) \in V$. 这表明

$$
f^{n}(U) \cap V \neq \emptyset, \quad f^{n}(V) \cap V \neq \emptyset,
$$

因此 $f$ 弱混合.

由定理 2.1 立刻有

推论 2.1 若 $f$ 混合或弱混合, 则 $\bar{f}$ 传递.

因为 $f$ 周期稠密蕴涵 $\bar{f}$ 周期稠密 ${ }^{[11]}$, 所以还有

推论 2.2 当 $f$ 混沌且弱混合时, $\bar{f}$ 混沌; 反之, 若 $\bar{f}$ 混沌, 则 $f$ 必弱混合. 
注 2.1 推论 2.2 是说仅当 $f$ 弱混合时, $f$ 混沌蕴涵 $\bar{f}$ 混沌的论断才成立, 这对 Fedeli 提出的问题给出了明确的回答.

定理 $2.2 \bar{f}$ 混合当且仅当 $f$ 混合.

证 必要性简单, 充分性用到引理 1.2 , 其证明类似于定理 2.1 (i) $\Rightarrow$ (ii), 故从 略.

注 2.2 当 $X$ 是任意拓扑空间 (相应地, $\mathcal{K}(X)$ 为赋予 Vietoris 拓扑的拓扑空 间) 时, 引理 $1.1 \sim 1.3$ 成立, 因而由证明过程可见定理 2.1 和 2.2 也成立.

注 2.3 文献 [13,14] 中刻画了混合映射和弱混合映射的复杂动力性态, 定 理 2.1 和 2.2 可以为已有的刻画增添新的内容.

\section{3 区间映射与 Román-Flores 问题的回答}

下文中， $I$ 表示单位闭区间 $[0,1]$.

引理 3.1 ${ }^{[15]}$ 若 $f: I \rightarrow I$ 为传递的连续映射, 则下述条件之一成立:

(1) $f$ 混合,

(2) 存在不动点 $e \in(0,1)$, 使得

$$
f([0, e])=[e, 1], \quad f([e, 1])=[0, e] .
$$

引理 3.2 设 $f: I \rightarrow I$ 为帐篷映射, 亦即 $f(x)=|1-2 x|$, 则 $f$ 是混合的.

证 如熟知, $f$ 传递 ${ }^{[3]}$. 注意到 $e=1 / 3$ 是 $f$ 仅有的不动点且 $f([e, 1])=I \neq$ $[0, e]$, 由引理 3.1, $f$ 混合.

命题 3.1 若 $f: I \rightarrow I$ 连续, 则下述条件等价:

(1) $f$ 混合,

(2) $f$ 弱混合,

(3) $\bar{f}$ 混沌,

(4) $\bar{f}$ 传递,

(5) $\bar{f}$ 混合,

(6) $\bar{f}$ 弱混合.

证 由定理 2.1 和 2.2, 只需证明 (1) $\Leftrightarrow$ (2) $\Leftrightarrow$ (3) 就可以了.

$(1) \Rightarrow(2)$. 显然.

$(2) \Rightarrow(3)$. 因为 $f$ 弱混合 $\Rightarrow f$ 传递 $\Rightarrow f$ 混沌 ${ }^{[16]}$, 据推论 $2.2, \bar{f}$ 混沌.

$(3) \Rightarrow(1)$. 设 $\bar{f}$ 混沌. 据推论 $2.2, f$ 弱混合, 进而 $f$ 传递. 若 $f$ 不是混合, 则由引理 3.1 知存在 $e \in(0,1)$, 使得 (3.1) 式成立. 令 $\varepsilon=\min \{e / 2,(1-e) / 2\}$. 考 察 $\mathcal{K}(X)$ 的开子集 $B(I, \varepsilon)$ 与 $B(\{1\}, \varepsilon)$. 若 $K \in B(\{1\}, \varepsilon)$, 则 $K \subset[e, 1]$, 因此对任 意 $n>0, f^{n}(K)$ 或含于 $[0, e]$, 或含于 $[e, 1]$. 无论怎样总有 $H\left(\bar{f}^{n}(K), I\right) \geqslant \varepsilon$. 于是 $\bar{f}^{n}(B(\{1\}, \varepsilon)) \cap B(I, \varepsilon)=\emptyset$, 进而 $\bar{f}$ 不是传递, 这与 $\bar{f}$ 是混沌的假设矛盾.

注 3.1 命题 3.1 中 (1) 和 (2) 等价是文献 [13] 中的已有结果, 但其余是新 的.

例 3.1 存在连续映射 $f: I \rightarrow I$, 使得 $f$ 混沌但 $\bar{f}$ 不混沌. 
令 $f: I \rightarrow I$ 定义为对任意 $x \in I$,

$$
f(x)= \begin{cases}2 x+\frac{1}{2}, & 0 \leqslant x \leqslant \frac{1}{4}, \\ \frac{3}{2}-2 x, & \frac{1}{4} \leqslant x \leqslant \frac{1}{2}, \\ 1-x, & \frac{1}{2} \leqslant x \leqslant 1,\end{cases}
$$

易见 $1 / 2$ 是 $f$ 的不动点且

$$
f([0,1 / 2])=[1 / 2,1], \quad f([1 / 2,1])=[0,1 / 2] .
$$

由于不难验证限制映射 $\left.f^{2}\right|_{[0,1 / 2]}$ 和 $\left.f^{2}\right|_{[1 / 2,1]}$ 都与 $I$ 上的帐㩐映射拓扑共轭, 因 而由引理 3.2, 它们都是混合的. 据文献 [13] 命题 3.3, $f$ 传递, 从而 $f$ 混沌 ${ }^{[16]}$. 明 显地, $f$ 不是混合, 因此由命题 $3.1, \bar{f}$ 不混沌.

注 3.2 例 3.1 对 Román-Flores 提出的问题给出了否定的回答.

\section{4 例子与问题}

例 4.1 设 $f$ 为帐篷映射. 由引理 3.2 和命题 3.1, $\bar{f}$ 混沌. 这是文献 $[10,11]$ 中同一结果的简单证明.

例 4.2 设 $\lambda$ 为一无理数, $T: S^{1} \rightarrow S^{1}$ 定义为 $T\left(\mathrm{e}^{\mathrm{i} x}\right)=\mathrm{e}^{\mathrm{i}(x+2 \pi \lambda)}$, 亦即 $T$ 是 复平面上单位圆周的无理旋转. 如熟知, $T$ 传递 ${ }^{[17]}$. 令 $(a, b)$ 表示 $S^{1}$ 上从 $a$ 沿逆 时针方向到 $b$ 的开弧. 考虑 $S^{1}$ 上的开弧 $U=\left(1, \mathrm{e}^{\mathrm{i} \pi / 4}\right)$ 和 $V=\left(-1, \mathrm{e}^{\mathrm{i} 5 \pi / 4}\right)$. 因为 $T$ 是等距, 故不难看出对任意 $n>0, T^{n}(U) \cap V \neq \emptyset$ 与 $T^{n}(V) \cap V \neq \emptyset$ 不能同时 成立, 因此 $T$ 不是弱混合, 进而由定理 2.1, $T$ 不传递. 这是文献 [10] 中同一结 果的简单证明.

例 4.3 设 $\Sigma=\left\{s=\left(s_{0}, s_{1}, s_{2}, \cdots\right) \mid s_{j}=0\right.$ 或 1$\}$ 为具有两个符号的符号空 间, 其中 $\Sigma$ 中任意两点 $s=\left(s_{0}, s_{1}, s_{2}, \cdots\right)$ 和 $t=\left(t_{0}, t_{1}, t_{2}, \cdots\right)$ 的距离定义为

$$
d(s, t)=\sum_{i=0}^{\infty} \frac{\left|s_{i}-t_{i}\right|}{2^{i}} .
$$

容易证明 $d(s, t) \leqslant 1 / 2^{n}$ 当且仅当对每个 $i=0,1, \cdots, n, s_{i}=t_{i}$. 令 $\sigma: \Sigma \rightarrow \Sigma$ 为移 位映射, $\sigma$ 连续而且混沌 ${ }^{[3]}$. 对任意非空开集 $U$, 存在 $n>0$, 使得 $\sigma^{n}(U)=\Sigma$, 因 此易见 $\sigma$ 是混合, 特别地是弱混合. 于是由推论 $2.2, \bar{\sigma}$ 混沌.

例 4.4 设 $\Sigma$ 为例 4.3 中的符号空间. 对 $\Sigma$ 中任意两点 $s=\left(s_{0}, s_{1}, s_{2}, \cdots\right)$ 和 $t=\left(t_{0}, t_{1}, t_{2}, \cdots\right)$, 加法

$$
s+t=\left(u_{0}, u_{1}, u_{2}, \cdots\right)
$$

定义为若 $s_{0}+t_{0}<2$, 则 $u_{0}=s_{0}+t_{0}$; 若 $s_{0}+t_{0} \geqslant 2$, 则 $u_{0}=s_{0}+t_{0}-2$, 且把 1 带 到下一位; 用同样方式依次确定 $u_{1}, u_{2}, \cdots$. 在此加法下, $\Sigma$ 为紧致拓扑群, 记作 $\Sigma_{+}$. 
令 $\tau: \Sigma_{+} \rightarrow \Sigma_{+}$定义为 $\tau(s)=s+\mathbf{1}$, 这里 $s \in \Sigma_{+}, \mathbf{1}=(1,0,0, \cdots) . \tau$ 连续且 无周期点 ${ }^{[17]}$. 设 $\mathcal{B}\left(U_{1}, \cdots, U_{m}\right)$ 是 $\mathcal{K}\left(\Sigma_{+}\right)$拓扑基中的任意成员. 对每个 $U_{i}$, 存在 $\Sigma_{+}$的拓扑基中成员 $V_{i}$, 亦即形如

$$
\left[s_{0}, \cdots, s_{k}\right]=\left\{t \in \Sigma_{+} \mid t_{i}=s_{i}, 0 \leqslant i \leqslant k\right\}
$$

的柱形, 使得 $V_{i} \subset U_{i}$. 令 $V=V_{1} \cup V_{2} \cup \cdots \cup V_{m}$. 由于每个 $V_{i}$ 都是 $\Sigma_{+}$的紧子 集, 因此易见 $V \in \mathcal{K}\left(\Sigma_{+}\right) \cap \mathcal{B}\left(U_{1}, \cdots, U_{n}\right)$. 又由于对每个 $V_{i}$, 存在 $n_{i}>0$, 使得 $\tau^{n_{i}}\left(V_{i}\right)=V_{i}$, 故若令 $n=n_{1} n_{2} \cdots n_{m}$, 则 $\tau^{n}(V)=V$. 所以 $V$ 是 $\bar{\tau}$ 的周期点, 从而 $\bar{\tau}$ 周期稠密.

注 4.1 例 4.4 表明 $\bar{f}$ 周期稠密并不意味 $f$ 也是如此, 这正是推论 2.2 中未 能包含 $\bar{f}$ 混沌蕴涵 $f$ 混沌论断的原因. 不过若注意到例 4.4 中的 $\bar{\tau}$ 并不是混沌的 (由于不传递), 可知下述问题依然存在:

\section{(Q3) $\bar{f}$ 混沌是否蕴涵 $f$ 混沌?}

注 4.2 除问题 (Q3) 外还有许多值得考虑的问题, 例如 $f$ 与 $\bar{f}$ 拓扑熵间的 关系; 传递性与弱混合性等价除 $\bar{f}$ 外还对哪些系统成立等. 我们希望这些问题 能够引起同行的兴趣.

\section{参考文献}

1 Ashwin P. Attractors of a randomly forced electronic oscillator. Physica D, 1999, 125: 302 310

2 Kaczynski T, Mrozek M. Conley index for discrete multivalued systems. Topology and its Applications, 1995, 65: 83 96

3 Devaney R. An Introduction to Chaotic Dynamical Systems. Redwood City: Addison-Wesley, 1989

4 Banks J, Brooks J, Cairns G, et al. On the definition of chaos. Amer Math Monthly, 1992, 99: 332 334

5 Silverman S. On maps with dense orbits and the definition of chaos. Rocky Mountain J Math, 1992, 22: 353 375

6 周作领. 符号动力系统. 上海: 上海科技教育出版社, 1997

7 Huang W, Ye X. Devaney's chaos or 2-scattering implies Li-Yorke's chaos. Topology and its Applications, 2002, 117: 259 272

8 Mai J. Devaney's chaos implies existence of s-scrambled sets. Proc Amer Math Soc, 2004, 132: 2761 2767

9 熊金城. 拓扑传递系统中的混沌. 中国科学, A 辑, 2005, 35(3): 252 261

10 Román-Flores H. A note on transitivity in set-valued discrete systems. Chaos, Solitons and Fractals, 2003, 17: 99 104

11 Fedeli A. On chaotic set-valued discrete dynamical systems. Chaos, Solitons and Fractals, 2005, 23: $1381 \sim 1384$

12 Klein E, Thompson A. Theory of Correspondences. New York: Wiley-Inter-Science, 1984

13 Xiong J, Yang Z. Chaos caused by a topologically mixing maps. In: Dynamical Systems and Related Topics. Singapore: World Scientific Press, 1992. 550 572

14 熊金城, 陈二才. 强混合的保测变换引起的混沌. 中国科学, A 辑, 1996, 26(11): 961 967

15 Barge M, Martin J. Chaos, periodicity and snakelike continua. Trans Amer Math Soc, 1985, 289: 355 365

16 Vellekoop M, Berglund R. On intervals, transitivity = chaos. Amer Math Monthly, 1994, 101: $353 \sim 355$

17 Block L S, Coppel W A. Dynamics in One Dimension. Lecture Notes in Math, Vol 1513. Berlin: SpringerVerlag, 1992 